This item was submitted to Loughborough's Research Repository by the author.

Items in Figshare are protected by copyright, with all rights reserved, unless otherwise indicated.

\title{
The influence of carbohydrate mouth rinse on self-selected intermittent running performance
}

\section{PLEASE CITE THE PUBLISHED VERSION}

http://journals.humankinetics.com/ijsnem-back-issues/ijsnem-volume-25-issue-6-december/the-influence-ofcarbohydrate-mouth-rinse-on-self-selected-intermittent-running-performance

\section{PUBLISHER}

(C) Human Kinetics as accepted for publication

\section{VERSION}

AM (Accepted Manuscript)

\section{PUBLISHER STATEMENT}

This work is made available according to the conditions of the Creative Commons Attribution-NonCommercialNoDerivatives 4.0 International (CC BY-NC-ND 4.0) licence. Full details of this licence are available at: https://creativecommons.org/licenses/by-nc-nd/4.0/

\section{LICENCE}

CC BY-NC-ND 4.0

\section{REPOSITORY RECORD}

Rollo, lan, George Homewood, Clyde Williams, James Carter, and Victoria L. Goosey-Tolfrey. 2019. "The Influence of Carbohydrate Mouth Rinse on Self-selected Intermittent Running Performance”. figshare. https://hdl.handle.net/2134/17550. 
1 THE INFLUENCE OF CARBOHYDRATE MOUTH RINSE ON SELF-SELECTED

2 INTERMITTENT RUNNING PERFORMANCE

3 Ian Rollo ${ }^{1}$, George Homewood ${ }^{2}$, Clyde Williams ${ }^{2}$, James Carter ${ }^{1}$, \& Vicky L. Goosey-

4 Tolfrey $^{2}$

5

$6 \quad{ }^{1}$ The Gatorade Sports Science Institute, PepsiCo, UK.

$7 \quad 2$ The School of Sport Exercise and Health Sciences, Loughborough University, UK.

8

9 Corresponding author:

10 Ian Rollo

11 The Gatorade Sports Science Institute

12 PepsiCo Corporate R\&D

13 Loughborough University

14 Leicestershire

15 UK

16 LE1 3TU

17 Ian.rollo@pepsico.com

18

19

20

Running Title: CARBOHYDRATE MOUTH RINSE AND VARIABLE INTENSITY RUNNING

21

22

23

24

25

26

27

28 


\section{ABSTRACT}

2 The present study investigated the influence of mouth rinsing a carbohydrate solution on self3 selected intermittent variable speed running performance. Eleven male soccer players 4 completed a modified version of the Loughborough Intermittent Shuttle Test (LIST) on two 5 occasions separated by 1 week. The modified LIST allowed the self-selection of running 6 speeds during block 6 of the protocol (75-90 min). Players rinsed and expectorated $25 \mathrm{ml}$ of

7 non-caloric placebo (PLA) or $10 \%$ maltodextrin solution $(\mathrm{CHO})$ for $10 \mathrm{~s}$, routinely during 8 block 6 of the LIST. Self-selected speeds during the walk and cruise phases of the LIST were 9 similar between trials. Jogging speed was significantly faster during the CHO $(11.3 \pm 0.7$ $\left.10 \mathrm{~km} \cdot \mathrm{h}^{-1}\right)$ than during the PLA trial $\left(10.5 \pm 1.3 \mathrm{~km} \cdot \mathrm{h}^{-1}\right)(\mathrm{P}=0.010) .15 \mathrm{~m}$ sprint speeds were 11 not different between trials (PLA: $2.69 \mathrm{~s} \pm 0.18 \mathrm{~s}$ : CHO: $2.65 \mathrm{~s} \pm 0.13 \mathrm{~s})(\mathrm{F}(2,10), \mathrm{P}=$ $120.157)$ but significant benefits were observed for sprint distance covered $(\mathrm{P}=0.024)$. The threshold for the smallest worthwhile change in sprint performance was set at $0.2 \mathrm{~s}$. Inferential statistical analysis showed the chance that $\mathrm{CHO}$ mouth rinse was beneficial, negligible or detrimental to repeated sprint performance was $86 \%, 10 \%$ and $4 \%$ respectively. In conclusion, mouth rinsing and expectorating a $10 \%$ maltodextrin solution was associated with a significant increase in self-selected jogging speed. Repeated $15 \mathrm{~m}$ sprint performance was also $86 \%$ likely to benefit from routinely mouth rinsing a carbohydrate solution in comparison to a taste matched placebo.

Word count: 246

Key Words 
1

2 The ingestion of well formulated carbohydrate-electrolyte (CHO-E) solutions have been 3

4

5

6

7

8

9

\section{INTRODUCTION}

reported to improve both continuous and intermittent running performance (Nicholas et al., 1995; Tsintzas \& Williams 1998). During prolonged exercise, carbohydrate (CHO) ingestion exerts its effect by maintaining blood glucose concentrations, maintaining $\mathrm{CHO}$ oxidation, and, under certain circumstances, delaying the depletion of liver and muscle glycogen.

However, some athletes are intolerant to ingesting CHO-E solutions and gels during exercise because of gastrointestinal distress (Brouns 1991). Furthermore, during prolonged variable high speed running the rate of gastric emptying is slowed, though it appears to adapt as exercise continues (Leiper et al., 2005).

Several recent studies have shown that simply mouth rinsing a $\mathrm{CHO}$ solution improves selfselected $1 \mathrm{~h}$ cycling time-trial and treadmill running performances (Carter et al., 2004; Chambers et al., 2009; Rollo et al., 2010; Lane et al., 2013). However, not all studies have found improvements in performance which may be due to several factors such as whether participants are fed or fasted, the duration and the concentration of the $\mathrm{CHO}$ rinse and the method of assessing exercise performance (Whitham \& McKinney 2007; Beelen et al., 2009; Sinclair et al., 2014). Nevertheless, when athletes are able to self-select their exercise intensities then the consensus view is that under some but not all exercise conditions mouth rinsing improves performance (Jeukendrup \& Chambers 2010; Rollo \& Williams 2011) (Burke \& Maughan 2014; de Ataide e Silva et al., 2014). One simple speculation about the positive influence of mouth rinsing is that $\mathrm{CHO}$ in the oral cavity is detected by the brain as an incoming supply of its essential fuel supply i.e. glucose. In the presence of this perceived impending increase in its fuel supply, the balance of excitation and inhibition of the brains motor cortex may be altered in favour of excitation, allowing athletes to increase their running speeds. 
1 In a recent study, mouth rinsing a 6.4\% maltodextrin solution was reported to have no benefit

2 on sprint running or repeated sprint performance of soccer players completing 4 blocks of the

3 Loughborough Intermittent Shuttle Running Test (LIST) (Dorling \& Earnest 2013).

4 However, performing this standard version of the LIST does not allow participants to self-

5 select running speeds, other than during the sprints. Recently, a modified form of the LIST

6 was introduced that includes a block of self-selected running speed that allows participants to

7 reduce their speed as they fatigue, as is the case during real-world competitions (Ali et al.,

8 2014). This more ecologically valid protocol has been used to tease out the performance

9 benefits of ingesting a $\mathrm{CHO}$ solution during prolonged intermittent high intensity running

10 (Highton et al., 2013).

11 Therefore, the aim of the present study was to investigate the influence of mouth rinsing a

12 CHO solution on "self-selected" variable speed running and repeated sprint performance.

METHOD

Participants

16 Eleven male soccer players gave their written consent before participating in this study 17 approved by the Loughborough University Ethical Advisory Committee. The players' 18 physiological characteristics are reported in Table 1. All players regularly ingested 19 carbohydrate-electrolyte solutions during training and when playing matches.

\section{Preliminary Measures}

21 All tests were completed in an indoor sports hall with a marked 20 m running track. Ambient conditions were maintained at $22.0 \pm 1.0^{\circ} \mathrm{C}$; relative humidity $32 \pm 2 \%$. During visit 1 each participant's $\dot{\mathrm{V}} \mathrm{O}_{2}$ max and velocity at $\dot{\mathrm{V}}{ }_{2}$ max was measured using a progressive multistage 
1 shuttle run test (Ramsbottom et al., 1988). This information was used to determine five

2 discrete pacing intervals to dictate the jogging $\left(55 \% \dot{\mathrm{V}} \mathrm{O}_{2} \max \right)$ and cruising $\left(95 \% \dot{\mathrm{V}} \mathrm{O}_{2} \mathrm{max}\right)$

3 speeds required for the experimental trials. Participants then completed a single 15-min block

4 of the Loughborough Intermittent Shuttle Test (LIST) (Figure 1) to familiarise themselves

5 with the exercise protocol (Nicholas et al., 2000). The participants also practiced mouth

6 rinsing with water during the final 3 cycles of activities.

7 Three psychological scales; Perceived Activation Scale (FAS) (Svebak \& Murgatroyd 1985),

$8 \quad$ Feeling Scale (FS) (Hardy \& Rejeski 1989) and Rating of Perceived Exertion Scale (RPE)

9 (Borg, 1982), were administered at rest and throughout exercise. Heart rate was recorded

10 every $5 \mathrm{~s}$ during the familiarisation and all experimental trials using short-range telemetry

11 (Polar Electro, UK).

12 Experimental Procedures

13 Study Design

14 The study employed a double blinded counter-balanced, cross-over design. Trials were 15 performed at the same time of day, separated by 1 week. The experimental solutions were 16 prepared and labelled by a non-affiliated research assistant. In the $24 \mathrm{~h}$ preceding each 17 experimental trial participants were asked to abstain from strenuous exercise, caffeine and 18 alcohol. On the morning of each trial participants were asked to consume a breakfast which 19 represented their usual match day meal $3 \mathrm{~h}$ before exercise.

20 Prior to both trials participants' body mass was recorded and their water bottles weighed.

21 Water intake was ad-libitum during the first 5 blocks of the LIST in the first experimental 22 trial. The volume ingested was then prescribed as the water intake during the second trial. All 23 but one participant completed the two experimental trials in pairs. The participants were 
1 matched based on their predicted $\dot{\mathrm{VO}}_{2}$ max scores from the progressive multistage shuttle run

2 test.

3 The LIST protocol is displayed (Figure 1). The run timing throughout the first five blocks of

4 the LIST was dictated by audio cues (Nicholas et al., 2000). The sixth and final stage of the

5 LIST was a self-selected stage without audio cues (Ali et al., 2014). Participants were

6 instructed to replicate the running routine of the five previous up to the completion of $90 \mathrm{~min}$.

7 Investigators gave no other verbal instruction during the self-paced section, apart from when

8 to cease exercise. During the self-paced section of the LIST participants were video recorded.

9 Analysis of the video provided an accurate record of the time spent (s) completing each activity, the number of LIST cycles completed and the stage of the LIST cycle that had been completed following the completion of $15 \mathrm{~min}$. The self-selected speed for each LIST activity was then calculated using the equation; distance/time. Sprint time was recorded over a distance of $15 \mathrm{~m}$ using infrared timing gates (Brower Timing Systems, USA).

\section{Carbohydrate Solutions and Procedure}

The carbohydrate $(\mathrm{CHO})$ solution was a $10 \%$ maltodextrin solution (MuscleTalk, Northamptonshire, UK). The placebo (PLA) solution was water. In an attempt to disguise the solutions both the CHO and PLA solutions were flavoured by an artificial non-caloric sweetener (FlavDrops, MyProtein UK). Each participant mouth rinsed the prescribed solution while performing the second of the three $20 \mathrm{~m}$ walks during the activity cycles in block 6 of the LIST (Figure 1). The mouth rinse procedure involved participants rinsing $25 \mathrm{ml}$ of experimental solution for $10 \mathrm{~s}$ before expectorating the solution into a pre-weighed container. This protocol resulted in the prescribed solutions being rinsed a total of 11 times during the final stage of the LIST. On completion of the trial, cups and containers were re-weighed to determine if any of the solution has been ingested. 
1

3

5

7

\section{RESULTS}

\section{Statistical Analysis}

Statistical analyses were performed using SPSS software package (version 18; SPSS inc., Chicago IL. USA). The Shapiro-Wilk test assessed the distribution of all data sets. In cases of non-normal distribution, the equivalent nonparametric statistical test was used. The mean differences in performance (walking, sprint, jogging, cruising speed, sprint number) were detected using a paired samples t-test. Changes in sprint times during block 6 of LIST were assessed using two-way (sprint number $\mathrm{x}$ condition) repeated measures ANOVA. The quantitative approach to likelihoods of benefit, triviality, and detrimental to running performance was further enriched by dividing the range of substantial values into more finely graded magnitudes. Using the spreadsheet by Hopkins (2007), the $P$ value was converted into $90 \%$ confidence intervals $(\mathrm{CI})$ for, and inferences about, the true value of the effect statistic (Hopkins 2007). The smallest worthwhile change in sprint time was set at $0.8 \%$ of the mean sprint time in both trials (Paton et al., 2001; Hopkins 2004). All results are reported as mean \pm standard deviation (SD) or $90 \%$ confidence intervals when appropriate. Statistical significance was set as $\mathrm{P}<0.10$.

\section{Running performance}

The self-selected distance covered and speed for each running occasion during the selfselected block 6 (75-90 $\mathrm{min})$ of the LIST is shown in Table 2. There was no trial order effect for all performance measures between trials $(\mathrm{P}>0.10$, Table 2).

There were no differences in the sprint times between CHO and PLA trials F $(2,10), \mathrm{P}=$ 0.157 (Figure 2). There was a small but significant increase in the number of $15 \mathrm{~m}$ sprints completed, Figure 3). In addition, participants maintained a significantly faster jogging speed 
1 in the final 15 min LIST block: $10.5 \pm 1.3 \mathrm{~km} \bullet \mathrm{h}^{-1}$ (PLA) vs. $11.3 \pm 0.7 \mathrm{~km} \bullet \mathrm{h}^{-1}$ (CHO). The

2 threshold value for the smallest worthwhile change in sprint time was set at $0.2 \mathrm{~s}(0.8 \%$ of

3 mean sprint time). Thus the chance that the value effect was beneficial, negligible or

4 detrimental to repeated sprint performance was $86 \%, 10 \%$ and $4 \%$ respectively.

$5 \quad$ Physiological Measures and Psychological Scales

6 Heart rate and psychological scores for the Feeling Scale, Felt Arousal Scale and Ratings of

7 Perceived Exertion during exercise are shown in Table 3. The mean volume of fluid

8 consumed during the first 5 blocks of the LIST was $1087 \pm 207 \mathrm{ml}(834 \mathrm{ml}-1403 \mathrm{ml})$. There

9 was no difference in the percentage of body mass lost over the duration of 90 min between

10 trials $1.3 \pm 0.5 \%(0.6-2.3 \%)$. The mean volume of expectorate for the PLA and CHO trials

11 was $23 \pm 2 \mathrm{ml}$ and $24 \pm 1 \mathrm{ml}$, respectively. Thus the difference between the volume rinsed and expectorated was $2 \pm 2 \mathrm{ml}$ in the PLA trial and $1 \pm 1 \mathrm{ml}$ in the CHO trial.

\section{Detection of Rinse solution}

14 Following the completion of the second experimental trial participants were asked two questions. First, if they could distinguish between experimental solutions and second in which of the two trials they believed they were mouth rinsing $\mathrm{CHO}$. Out of the 11 players none were able to distinguish between the two test solutions. However, when asked which trial they believed they were mouth rinsing $\mathrm{CHO}, 6$ players correctly identified the $\mathrm{CHO}$ mouth rinse trial (i.e. more than would be predicted by chance). Of these six, only 2 had better jogging and sprint performance in the $\mathrm{CHO}$ trial.

\section{DISCUSSION}

The main finding of this study was that mouth rinsing and expectorating a $10 \%$ maltodextrin solution significantly increased self-selected jogging speed during 75-90 min of variable 
1 intensity running. Furthermore, mouth rinsing with $\mathrm{CHO}$ was associated with an $86 \%$

2 likelihood of being beneficial to repeated $15 \mathrm{~m}$ sprint speed, with small but significant effect

3 on sprint distance covered. To our knowledge this was the first study to investigate the

4 influence of $\mathrm{CHO}$ mouth rinse on self-selected variable intensity running performance.

5 The results of the present study are consistent with previous running studies which have 6 reported the routine mouth rinse of a $\mathrm{CHO}$ solution increases self-selected submaximal 7 running speeds (Rollo et al., 2008; Rollo et al., 2010). However, these previous studies 8 involved continuous treadmill running for $30 \mathrm{~min}$ and $1 \mathrm{~h}$ and are therefore not specific to 9 demands of intermittent variable speed running and maximal sprints. To date, there have been few studies conducted on the influence of $\mathrm{CHO}$ mouth rinsing on performance during intermittent exercise, of variable intensity, or sprinting.

Dorling and Earnest (2013) reported that sprint and repeated sprint ability was not improved when games players mouth rinsed a $6.4 \%$ maltodextrin or flavour matched placebo during the LIST. In this study, male games players completed four blocks of the LIST, identical to that of the present study, up to the completion of $60 \mathrm{~min}$. The authors reported no difference in 20 m sprint performance throughout exercise or repeated sprint ability $(4$ x $20 \mathrm{~m}$ sprint; $20 \mathrm{~s}$ recovery) performed at 0,30 and $60 \mathrm{~min}$. To our knowledge this the only study to have investigated $\mathrm{CHO}$ mouth rinse and repeated sprint running performance. That Dorling and Earnest (2013) observed no differences in performance may possibly be a consequence of several key aspects of their protocol which differed from the present study. The exercise duration was $90 \mathrm{~min}$ in the present study compared to $60 \mathrm{~min}$ in the Dorling and Earnest (2013). It is not unreasonable to suggest that the reduction in muscle glycogen concentration was probably greater in the present study (Nicholas et al., 1999). The participants also reported increased perception of effort and detriment in 'feeling scale' following block five of the LIST (Table 3).Perceptions of greater effort during the latter stages of prolonged 
1 exercise are commonly associated with a decrease in $\mathrm{CHO}$ availability (Jeukendrup et al.,

2 2006). As such, it may be speculated that mouth rinsing a $\mathrm{CHO}$ solution during the last 15

3 min of a 90 min LIST may have had a greater impact than if administered earlier in exercise,

4 i.e. when participants are likely to have a greater endogenous store of $\mathrm{CHO}$.

5 The limitation of the traditional LIST protocol is that, other than the $20 \mathrm{~m}$ sprints, running 6 speeds are dictated by audio cues. Previous studies have shown that protocols which do not 7 allow "self-selected" exercise may not be sufficiently sensitive to detecting subtle alterations 8 in running speed in response to a CHO mouth rinse intervention (Rollo \& Williams 2009; 9 Rollo et al., 2010). The modified LIST protocol allowed participants to self-select their speed during the last $15 \mathrm{~min}$ of exercise (Ali et al., 2014). As a result, small but significant performance benefits were observed in response to mouth rinsing $\mathrm{CHO}$ (Table 2).

The mechanism(s) by which mouth rinsing with a CHO solution increased jogging speed and sprint performance during variable intensity running are unknown. A consequence of the mouth rinse and expectorate procedure is the absence of substrate delivery to the systemic circulation. Therefore, it is reasonable to speculate that the exposure of $\mathrm{CHO}$ to the oral cavity had a "central" effect. In support of this hypothesis, it is known that humans have the ability to "taste" glucose polymers (maltodextrin) (Lapis et al., 2014). In addition, studies have reported that mouth rinsing $\mathrm{CHO}$ is associated with the activation of reward centres in the brain (Chambers et al., 2009) and improved sensory perception (Turner et al., 2014). We have previous speculated that potential corresponding feelings of pleasure or perceived activation may manifest as altered exercise behaviour (increased running speed) (Rollo et al., 2008). However, the present study found no differences in perceived exertion or any psychological measures during exercise between trials (Table 3). Thus, an alternate explanation may be that towards the end of prolonged intermittent running the $\mathrm{CHO}$ rinse may alter the excitation and inhibition balance of centrally mediated motor output, which 
1 resulted in improved running performance (Gant et al., 2010). However, many of the studies

2 designed to provide explanations for the favourable influence of $\mathrm{CHO}$ mouth rinse on exercise performance have either been conducted at rest or in single limb exercise. Further

4 research is needed to ascertain if results from these studies also translate to whole body 5 exercise.

6 These speculations are largely based on the hypothesis that mouth rinsing a CHO solution 7 offers the "promise" of incoming energy to the brain when liver and muscle glycogen stores 8 are reduced by exercise. However, this theory does not explain improvements in peak power, 9 during sprint cycling, following pre-exercise mouth rinsing a carbohydrate solution reported by some (Beaven et al., 2013; Chong et al., 2014; Phillips et al., 2014) but not others (Chong et al., 2011) or reducing the fall in muscle torque during several isometric leg extensions contractions (Jensen et al., 2014). It is relevant to note that CHO mouth rinse has been reported to increase the activation of cortico-motor pathways and voluntary force production in both 'fresh' and fatigued muscle (Gant et al., 2010). Therefore the mouth rinse phenomenon may not simply be a consequence of promising the brain incoming energy during exercise that involves a significant demand on endogenous $\mathrm{CHO}$ stores.

17 A limitation of the present study is that it is unknown whether a full familiarisation would have impacted on subsequent pacing strategy employed by the participants during the main trials. The present study replicated the protocol of Ali et al., (2014). In this study the investigators did not include a full familiarisation in the preliminary measurements. Instead participants completed two blocks of the list, one prescribed and one self-paced. The present study used a single block of the LIST in the familiarisation, to introduce the players to the pattern of running and rinse procedure. As recommended by Ali et al. (2014) the present study utilised randomised counterbalanced design, which was sufficient to prevent a trial order effect (Table 2). Finally, as the experimental trials were ran in pairs it is unknown how 
1 the pacing of one player may have influenced the performance of the other. Future studies

2 may consider placing a partition between runners, staggered starts or individual performance

3 trials.

\section{$4 \quad$ Practical Implications}

5 The ability to measure self-selected running at a range of intensities under laboratory

6 conditions is of relevance to team "stop-and-go" sports. Although, players are unlikely to

7 repeatedly rinse $\mathrm{CHO}$ during a match, the findings of the present study suggest that when

$8 \mathrm{CHO}$ solutions are ingested late in exercise, performance benefits may be gained before the

9 substrate reaches the systemic circulation. Several studies report that both elite and sub-elite

10 football players' ability to perform high-intensity exercise is reduced in the final 15 min of a

1190 min game (Bangsbo 2014). Exposing the mouth to CHO mouth rinse may offer an acute

12 performance benefit, both in terms of faster 'recovery' speeds but also in small improvements

13 in sprinting in this critical period of a game (Mohr et al., 2003; Mohr et al., 2005).

14 In conclusion, mouth rinsing a 10\% maltodextrin solution was associated with increased selfselected jogging speed between 75 and 90 min of variable intensity running. Repeated $15 \mathrm{~m}$ sprint performance was also $86 \%$ likely to benefit from routinely mouth rinsing a carbohydrate solution in comparison to a taste matched placebo. These findings add to the evidence that mechanisms independent of $\mathrm{CHO}$ delivery to the circulation may influence exercise performance. 


\section{Disclaimer}

2 Ian Rollo \& James Carter are employees of the Gatorade Sports Science Institute, a division

3 of PepsiCo, Inc. The views expressed in this manuscript are those of the authors and do not

4 necessarily reflect the position or policy of PepsiCo Inc.

5

\section{Acknowledgements}

7 The study was designed by IR, GH, and VT; data were collected and analyzed by GH and IR,

8 data interpretation and manuscript preparation were undertaken by IR, CW and JC. All

9 authors approved the final version of the paper. Thank you to the England and Wales Cricket

10 Board (Disability Cricket) for proving funding to support the scholarship of GH. 
1 Table 1. Physiological Characteristics of the soccer players ( $n=11$, mean \pm SD).

\section{Characteristic}

Age (years)

Height (m)

Weight (kg)

Estimated $\dot{\mathrm{V}}_{2} \max \left(\mathrm{ml} \cdot \mathrm{kg}^{-1} \cdot \mathrm{min}^{-1}\right)$

Soccer Experience (years)

Training and/or matches per week

\section{Measure}

$22 \pm 3$

$1.78 \pm 0.10$

$75.0 \pm 7.6$

$54.0 \pm 3.0$

$13 \pm 5$

$1-4$ 
1 Figure 1. Schematic of study experimental protocol. Light shaded blocks represent audio dictated running speeds. Dark shaded block 6 2 represents self-selected speeds

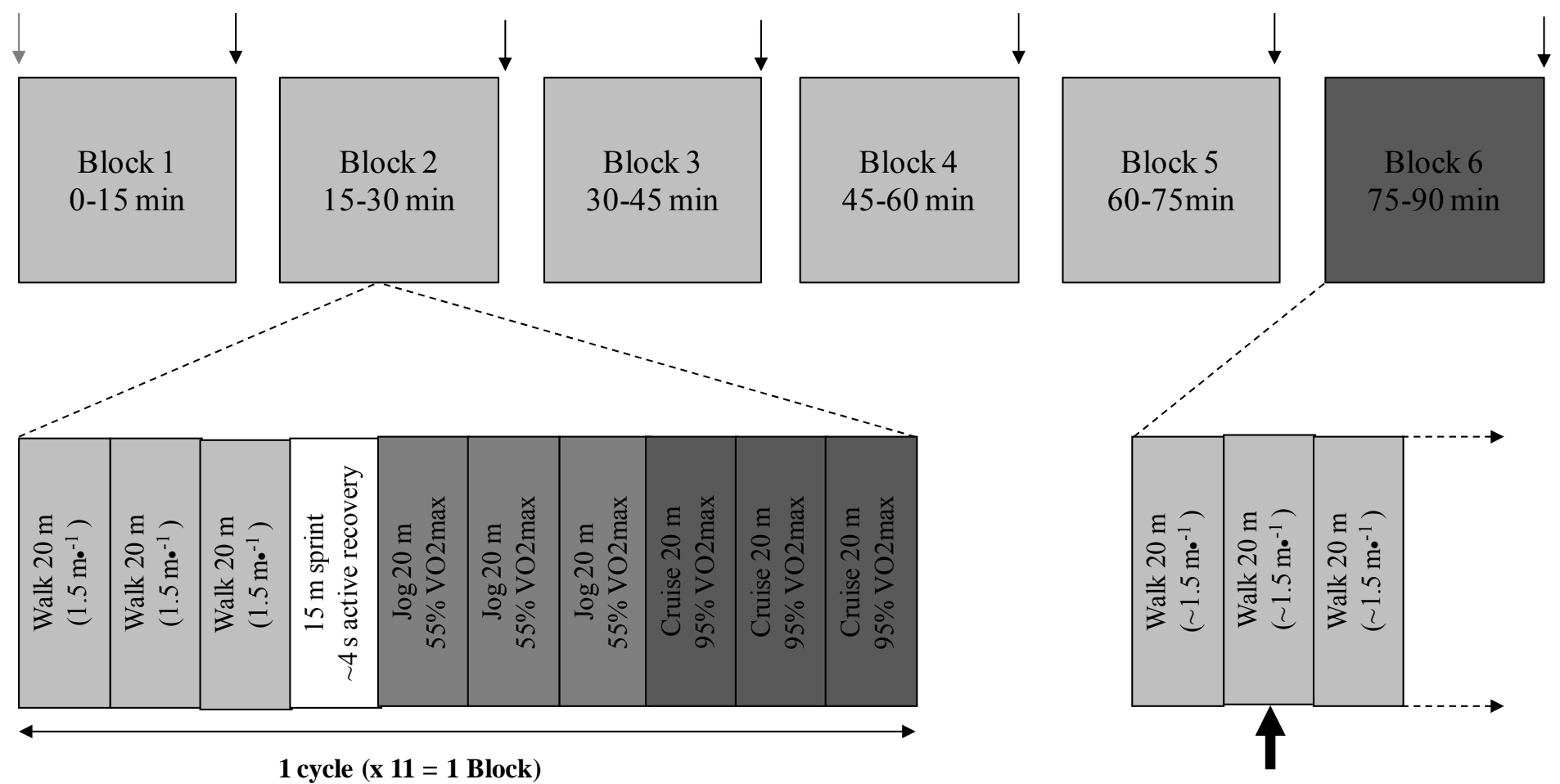

Key

FS, FAS

FS, FAS, RPE

Mouth Rinse 
1 Table 2. Self-selected running performance during block 6 (75-90 min) of the LIST, * indicates significant difference between trials.

\begin{tabular}{|c|c|c|c|c|c|c|}
\hline \multirow[t]{2}{*}{ Variable } & \multirow[t]{2}{*}{ PLA } & \multirow[t]{2}{*}{ CHO } & \multicolumn{2}{|c|}{$90 \% \mathrm{CI}$} & \multirow[t]{2}{*}{$\boldsymbol{P}$} & \multirow{2}{*}{$\begin{array}{c}\text { Trial Ordei } \\
P\end{array}$} \\
\hline & & & Lower & Upper & & \\
\hline Distance covered $(\mathrm{km})$ & $2.0 \pm 0.1$ & $2.0 \pm 0.0$ & -0.8 & 0.3 & 0.494 & 0.601 \\
\hline Mean speed $\left(\mathrm{km} \bullet \mathrm{h}^{-1}\right)$ & $8.7 \pm 0.5$ & $8.8 \pm 0.1$ & -0.3 & 0.1 & 0.513 & 0.563 \\
\hline \multicolumn{7}{|l|}{ Walking } \\
\hline Distance covered $(\mathrm{km})$ & $0.65 \pm 0.4$ & $0.65 \pm 0.2$ & -0.2 & 0.1 & 0.831 & 0.518 \\
\hline Mean speed $\left(\mathrm{km} \bullet \mathrm{h}^{-1}\right)$ & $5.5 \pm 0.2$ & $5.5 \pm 0.3$ & -0.1 & 0.3 & 0.419 & 0.930 \\
\hline \multicolumn{7}{|l|}{15 m Sprint } \\
\hline 15 m sprint time (s) & $2.69 \pm 0.18$ & $2.65 \pm 0.13$ & -0.2 & 0.10 & 0.298 & 0.839 \\
\hline Distance covered (km) & $0.15 \pm 012$ & $0.16 \pm 0.10$ & -0.0 & -0.00 & $0.024 *$ & 0.676 \\
\hline Mean speed $\left(\mathrm{km} \bullet \mathrm{h}^{-1}\right)$ & $20.1 \pm 1.2$ & $20.4 \pm 1.0$ & -0.8 & 0.2 & 0.316 & 0.855 \\
\hline \multicolumn{7}{|l|}{ Jogging } \\
\hline Distance covered $(\mathrm{km})$ & $0.60 \pm 0.0$ & $0.61 \pm 0.0$ & -0.0 & -0.0 & 0.450 & 0.653 \\
\hline Mean speed $\left(\mathrm{km} \bullet \mathrm{h}^{-1}\right)$ & $10.5 \pm 1.3$ & $11.3 \pm 0.7$ & -1.3 & -0.3 & $0.010^{*}$ & 0.549 \\
\hline \multicolumn{7}{|l|}{ Cruising } \\
\hline Distance covered $(\mathrm{km})$ & $0.6 \pm 0.4$ & $0.6 \pm 0.0$ & -0.0 & 0.0 & 0.676 & 0.676 \\
\hline Mean speed $\left(\mathrm{km} \bullet \mathrm{h}^{-1}\right)$ & $12.7 \pm 1.0$ & $12.8 \pm 0.9$ & -0.4 & 0.3 & 0.896 & 0.828 \\
\hline \multicolumn{7}{|l|}{ Recovery Time } \\
\hline Total (s) & $78 \pm 22$ & $76 \pm 13$ & -4.0 & 7.0 & 0.625 & 0.894 \\
\hline Mean time (s) & $7.8 \pm 2.6$ & $7.2 \pm 1.6$ & -0.1 & 1.2 & 0.171 & 1.000 \\
\hline
\end{tabular}


1 Figure 2. Mean and individual sprint times (s) during block 6 of LIST for PLA and CHO mouth rinse trials.

2

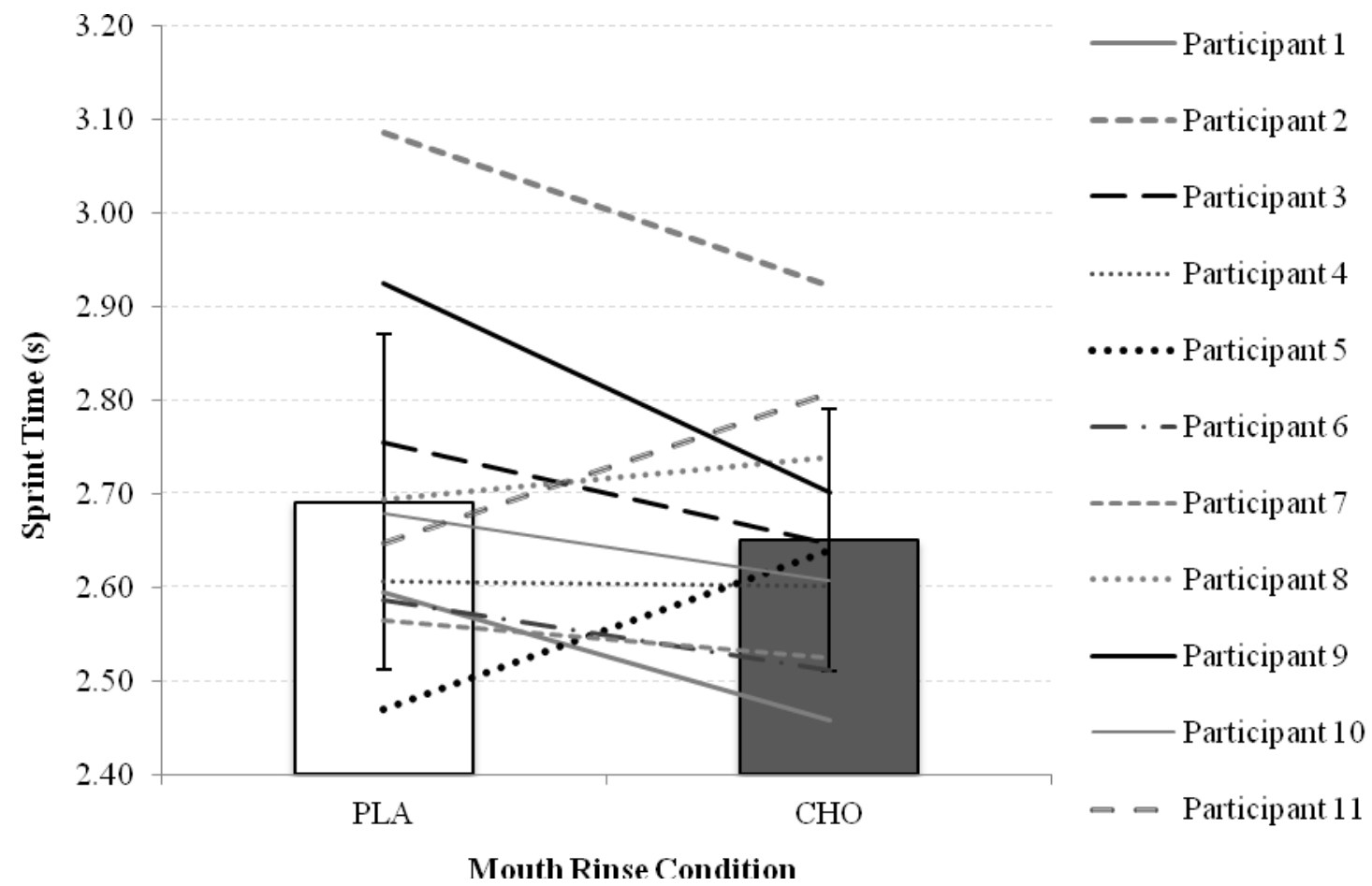


1 Table 3. Heart rate and psychological scale scores for Rating of Perceived Exertion (RPE), Felt Arousal Scale and Feeling Scale during each

2 block of the LIST. ${ }^{\#}$ Denotes a significant difference over time i.e. between blocks.

\begin{tabular}{|c|c|c|c|c|c|c|c|}
\hline Measure & Rest & Block 1 & Block 2 & Block 3 & Block 4 & Block 5 & Block 6 \\
\hline \multicolumn{8}{|c|}{ Heat rate (beat•min ${ }^{-1)}$} \\
\hline PLA & $100 \pm 10$ & $165 \pm 14 \#$ & $170 \pm 12$ & $170 \pm 11$ & $169 \pm 11$ & $167 \pm 13$ & $163 \pm 13$ \\
\hline $\mathrm{CHO}$ & $101 \pm 10$ & $167 \pm 15 \#$ & $168 \pm 12$ & $167 \pm 12$ & $167 \pm 15$ & $168 \pm 12$ & $165 \pm 12$ \\
\hline \multicolumn{8}{|l|}{ RPE } \\
\hline PLA & $\mathrm{n} / \mathrm{a}$ & $12.4 \pm 1.1$ & $13.6 \pm 1.2 \#$ & $14.4 \pm 1.8 \#$ & $15.2 \pm 1.6 \#$ & $16.2 \pm 1.7 \#$ & $16.4 \pm 1.8$ \\
\hline $\mathrm{CHO}$ & $\mathrm{n} / \mathrm{a}$ & $12.0 \pm 1.4$ & $13.5 \pm 1.6 \#$ & $14.4 \pm 1.5 \#$ & $15.4 \pm 1.9 \#$ & $16.4 \pm 2.0 \#$ & $16.3 \pm 2.2$ \\
\hline \multicolumn{8}{|c|}{ Felt Arousal Scale } \\
\hline PLA & $2.2 \pm 0.9$ & $2.9 \pm 1.0$ & $3.1 \pm 1,0$ & $3.0 \pm 0.9$ & $2.8 \pm 1.3$ & $2.9 \pm 1.4$ & $3.0 \pm 1.4$ \\
\hline $\mathrm{CHO}$ & $2.9 \pm 0.9$ & $3.4 \pm 1.0$ & $3.3 \pm 1.4$ & $2.8 \pm 1.5$ & $2.8 \pm 1.4$ & $2.4 \pm 1.7$ & $2.7 \pm 1.4$ \\
\hline \multicolumn{8}{|c|}{ Feeling scale } \\
\hline PLA & $2.5 \pm 1.7$ & $1.0 \pm 0.8 \#$ & $0.7 \pm 1.2$ & $0.9 \pm 1.6$ & $-1.0 \pm 1.7 \#$ & $-1.5 \pm 2.2$ & $-1.2 \pm 2.0$ \\
\hline $\mathrm{CHO}$ & $2.6 \pm 0.9$ & $1.8 \pm 0.8 \#$ & $0.5 \pm 1.7$ & $0.2 \pm 2.1$ & $-1.0 \pm 2.0 \#$ & $-1.5 \pm 2.6$ & $-1.2 \pm 3.2$ \\
\hline
\end{tabular}

3 
1 Figure 3. Mean number of sprints completed during block 6 of LIST for PLA and CHO mouth rinse trials. The lines "a" to "d""present the 2 number of players who either maintained or increased the number of sprints completed $(\mathrm{P}=0.016)$.

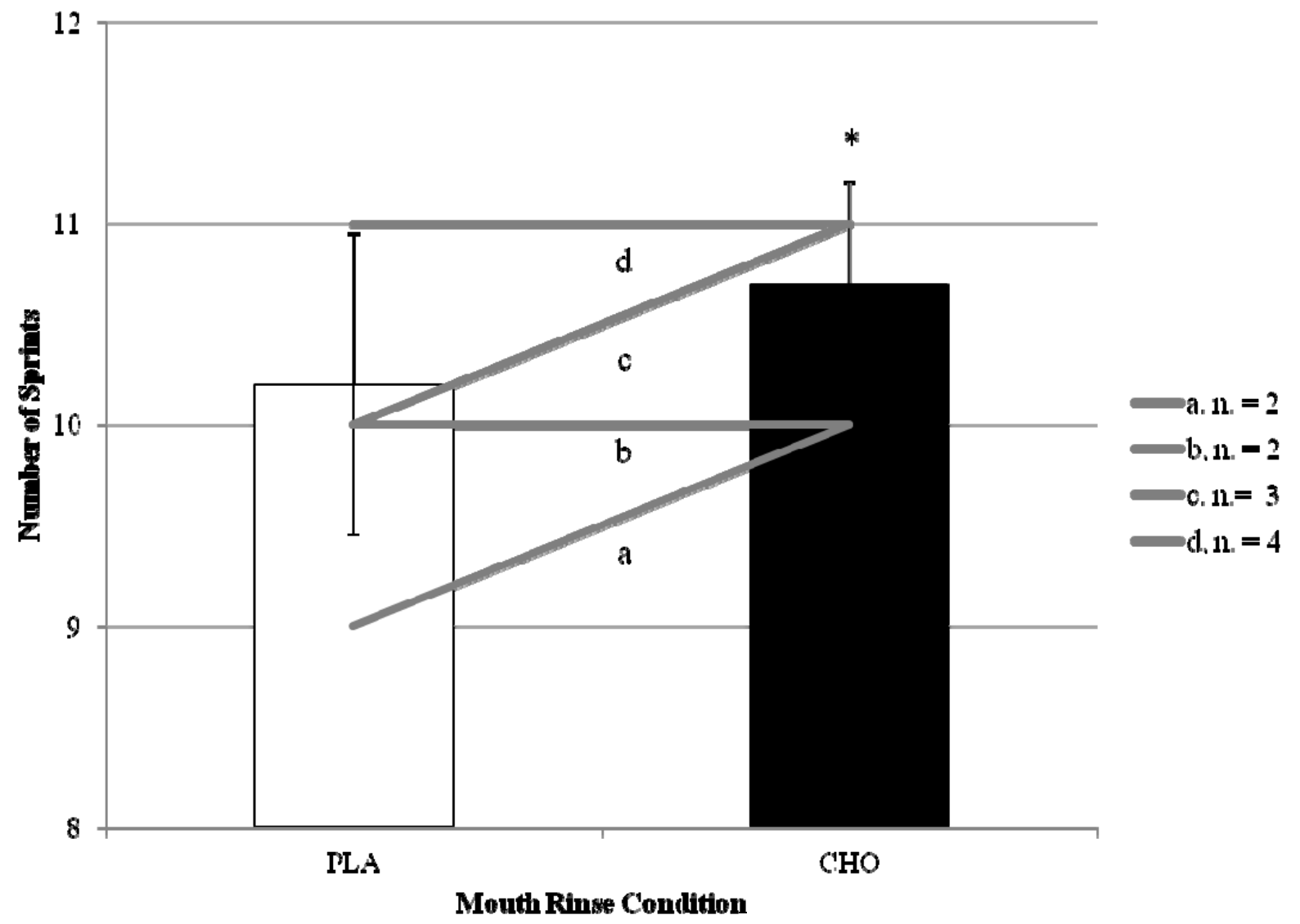




\section{REFERENCES}

2 Ali, A., A. Foskett \& N. Gant (2014). Measuring intermittent exercise performance using

3 shuttle running. Journal of Sports Sciences, 32(7): 601-609.

4 Bangsbo, J. (2014). Physiological Demands of Football. Gatorade Sports Science Institute, 5 Sports Science Exchange Article, 27(125): 1-6.

6 Beelen, M., J. Berghuis, B. Bonaparte, S. Ballak, A. E. Jeukendrup \& L. J. C. van Loon

7 (2009). Carbohydrate mouth rinsing in the fed state does not enhance time trial performance.

8 International Journal of Sports Nutrition and Exercise Metabolism, 19(4): 400-409.

9 Beaven, C. M., P. Maulder, A. Pooley, L. Kilduff \& C. Cook (2013). Effects of caffeine and 10 carbohydrate mouth rinses on repeated sprint performance. Appl Physiol Nutr Metab 38(6): $11 \quad 633-637$.

12 Borg, G. (1982). Ratings of perceived exertion and heart rates during short-term cycle exercise and their use in a new cycling strength test. International Journal of Sports

14 Medicine, 3(3), 153-158.

Brouns, F. (1991). Etiology of gastrointestinal disturbances during endurance events. Scandinavian Journal of Medicine \& Science in Sports, 1: 66-77.

17 Burke, L. M. \& R. J. Maughan (2014). The Governor has a sweet tooth - Mouth sensing of nutrients to enhance sports performance. European Journal of Sport Sciences, 27: 1-12.

Carter, J. M., A. E. Jeukendrup \& D. A. Jones (2004). The effect of carbohydrate mouth rinse on 1-h cycle time trial performance. Medicine and Science in Sports and Exercise, 36(12): 2107-2111.

Chambers, E. S., M. W. Bridge \& D. A. Jones (2009). Carbohydrate sensing in the human mouth: effects on exercise performance and brain activity. Journal of Physiology, 578.8: 1779-1794.

Chong, E., K. J. Guelfi \& P. A. Fournier (2011). Effect of a carbohydrate mouth rinse on maximal sprint performance in competitive male cyclists. Journal of Science and Medicine in Sport, 14(2): 162-167. 
1 Chong, E., K. J. Guelfi \& P. A. Fournier (2014). Combined Glucose Ingestion and Mouth

2 Rinsing Improves Sprint Cycling Performance. International Journal of Sport Nutrition and

3 Exercise Metabolism, 24(6): 605-612.

4 de Ataide e Silva, T., M. E. Di Cavalcanti Alves de Souza, J. F. de Amorim, C. G. Stathis, C.

5 G. Leandro \& A. E. Lima-Silva (2014). Can carbohydrate mouth rinse improve performance

6 during exercise? A systematic review. Nutrients, 6(1): 1-10.

7 Dorling, J. L. \& C. P. Earnest (2013). Effect of carbohydrate mouth rinsing on multiple sprint 8 performance. Journal of International Society of Sports Nutrition, 10(1): 41.

9 Gant, N., C. M. Stinear \& W. D. Byblow (2010). Carbohydrate in the mouth immediately 10 facilitates motor output. Brain Research, 1350: 151-158.

11 Hardy, C., J \& W. Rejeski (1989). Not what, but how ones feels: the measurement of affect 12 during exercise. J. Sport Exerc. Psychol 11: 304-317.

13 Highton, J., C. Twist, K. Lamb \& C. Nicholas (2013). Carbohydrate-protein coingestion 14 improves multiple-sprint running performance. Journal of Sports Sciences, 31(4): 361-369.

15 Hopkins, W. G. (2004). How to interpret changes in athletic performance test. Sportscience, $168(1-7)$.

17 Hopkins, W. G. (2007). A spreadsheet for deriving a confidence interval, mechanistic inference and clinical inference from a $\mathrm{p}$ value. Sportscience, 11: 16-20. Jensen, M., T. Stellingwerff \& M. Klimstra (2014). Carbohydrate Mouth rinse Counters 20 Fatigue Related Strength Reduction. International Journal of Sport Nutrition and Exercise Metabolism. E-publication ahead of print.

22 Jeukendrup, A. E. \& E. S. Chambers (2010). Oral carbohydrate sensing and exercise 23 performance. Current Opinion in Clinical Nutrition and Metabolism Care, 13(4): 447-451. Jeukendrup, A. E., L. Moseley, G. I. Mainwaring, S. Samuels, S. Perry \& C. H. Mann (2006). Exogenous carbohydrate oxidation during ultraendurance exercise. Journal of Applied Physiology, 100(4): 1134-1141. 
1 Lane, S. C., S. R. Bird, L. M. Burke \& J. A. Hawley (2013). Effect of a carbohydrate mouth

2 rinse on simulated cycling time-trial performance commenced in a fed or fasted state.

3 Applied Physiology and Nutrition Metabolism 38(2): 134-139.

4 Lapis, T. J., M. H. Penner \& J. Lim (2014). Evidence that Humans Can Taste Glucose 5 Polymers. Chemical Senses, 39(9): 737-747.

6 Leiper, J., C. Nicholas, A. Ali, C. Williams \& R. Maughan (2005). The effect of intermittent 7 high intensity running on gastric emptying of fluids in man. Medicine and Science Sports and $8 \quad$ Exercise. 37: 240-247.

9 Mohr, M., P. Krustrup \& J. Bangsbo (2003). Match performance of high-standard soccer 10 players with special reference to development of fatigue. Journal of Sports Sciences, 21(7): $11 \quad 519-528$.

Mohr, M., P. Krustrup \& J. Bangsbo (2005). Fatigue in soccer: a brief review. Journal of Sports Sciences, 23(6): 593-599.

Nicholas, C., C. Williams, L. Boobis \& N. Little (1999). Effect of ingesting a carbohydrateelectrolyte beverage on muscle glycogen utilisation during high intensity, intermittent shuttle running. Medicine and Science Sports and Exercise, 31: 1280-1286.

Nicholas, C., C. Williams, H. Lakomy, G. Phillips \& A. Nowitz (1995). Influence of ingesting a carbohydrate-electrolyte solution on endurance capacity during intermittent, high intensity shuttle running. Journal of Sports Sciences, 13: 283-290.

Nicholas, C. W., F. E. Nuttall \& C. Williams (2000). The Loughborough Intermittent Shuttle Test: a field test that simulates the activity pattern of soccer. Journal of Sports Sciences, 18(2): 97-104.

Paton, C. D., W. G. Hopkins \& L. Vollebregt (2001). Little effect of caffeine ingestion on repeated sprints in team-sport athletes. Medicine and Science Sports and Exercise, 33(5): 822-825.

Phillips, S. M., S. Findlay, M. Kavaliauskas \& M. C. Grant (2014). The Influence of Serial

Science and Medicine, 13(2): 252-258. 
1 Ramsbottom, R., J. Brewer \& C. Williams (1988). A progressive shuttle run test to estimate maximal oxygen uptake. British Journal of Sports Medicine, 22(4): 141-144.

3 Rollo, I., M. Cole, R. Miller \& C. Williams (2010). The Influence of Mouth-Rinsing A 4 Carbohydrate Solution on 1 Hour Running Performance. Medicine \& Science in Sports \& 5 Exercise 42(4): 798-804.

6 Rollo, I. \& C. Williams (2009). Influence of Ingesting a Carbohydrate-Electrolyte Solution

7 Before and During a 1-hr Running Performance Test. International Journal of Sports

$8 \quad$ Nutrition and Exercise Metabolism 19(6): 645-658.

9 Rollo, I. \& C. Williams (2011). Effect of mouth-rinsing carbohydrate solutions on endurance 10 performance. Sports Medicine, 41(6): 449-461.

11 Rollo, I., C. Williams, N. Gant \& M. Nute (2008). The Influence of Carbohydrate Mouth 12 Rinse on Self-Selected Speeds during a 30-min Treadmill Run. International Journal of 13 Sports Nutrition and Exercise Metabolism, 18(6): 585-600.

Sinclair, J., L. Bottoms, C. Flynn, E. Bradley, G. Alexander, S. McCullagh, T. Finn \& H. T. Hurst (2014). The effect of different durations of carbohydrate mouth rinse on cycling performance. European Journal of Sport Science, 14(3): 259-264.

Svebak, S. \& S. Murgatroyd (1985). Metamotivational dominance: a multimethod validation of reversal theory constructs. Journal of Personality and Social Psychology 48: 107-116. Tsintzas, K. \& C. Williams (1998). Human muscle glycogen metabolism during exercise: effect of carbohydrate supplementation. Sports Medicine, 25: 7-23. enhances activation of brain circuitry involved in motor performance and sensory perception. Appetite, 80: 212-219. Whitham, M. \& J. McKinney (2007). Effect of a carbohydrate mouthwash on running timetrial performance. Journal of Sports Sciences, 25(12): 1385-1392. 